\title{
Dynamic Lighting in Classrooms: A new interactive tool for teaching
}

\author{
Kathrine Schledermann ${ }^{1}$, Henrika Pihlajaniemi ${ }^{2,1}$, Sumit Sen ${ }^{1}$ and \\ Ellen Kathrine Hansen ${ }^{1}$ \\ ${ }^{1}$ Lighting Design, Department of Architecture, Design and Media Technology. \\ Aalborg University, A. C. Meyers Vænge 15, 2450 Copenhagen SV, Denmark \\ ekh@create.aau.dk, kathrine@schleder.dk, sumit.sen571@gmail.com \\ ${ }^{2}$ Oulu School of Architecture, University of Oulu. Pentti Kaiteran katu 1, 90014 Oulu, Finland \\ henrika.pihlajaniemi@oulu.fi
}

\begin{abstract}
This paper presents the results of a field study on the use of lighting as a tool to structure and support teaching and learning activities by teachers. In a Danish elementary school, a dynamic lighting technology with the option of choosing four different lighting scenarios was installed in classrooms. The teachers' use of the lighting was studied combining qualitative research material from observations and interviews and quantitative data from the lighting control system which indicated the teachers' choices for lighting scenarios. As a result, different types of motivations for interacting with lighting emerged in the analysis: Supporting and structuring learning activities; Communicating with lighting and involving students; Affecting students activity level and behavior; Creating atmosphere; and Supporting visual task and visual comfort. All these motivational aspects should be considered when designing dynamic lighting systems for learning environments, thus creating a new interactive tool for teachers.
\end{abstract}

Keywords: Interactive lighting, dynamic lighting, lighting design, learning environment, teaching tool, field study, interaction, user-centric design

\section{$1 \quad$ Introduction}

According to the Danish Ministry of Education, students will have spent 13.000 hours at school by the end of the $9^{\text {th }}$ grade [1], most of which in classrooms designed before the development of dynamic lighting technologies and digital devices such as smart boards and IPads. Neither the classrooms nor their lighting has been designed for the rapidly changing activities entailed by the different pedagogical approaches. Although this indicates the potential for tailor-made lighting scenarios for various learning situations, there still is a lack of knowledge on how teachers will adopt these dynamic lighting applications as interactive tools for teaching. 


\subsection{Light for Learning}

Previous studies conclude that long-term exposure to blue-enriched light especially during morning lessons increases the students' academic performance, concentration and progression $[3,4,5,6,7,8]$. However, the studies using academic performance as a test parameter showed a contradiction between findings from field studies and the controlled experiments [2]. Other studies indicate that exposure to warm light can reduce aggression and positively affect social behavior $[7,8,9]$. The review points out the need to focus on users' needs through more holistic field research approach. Therefore, this field study uses a holistic methodology where several factors and the relation between them are studied to indicate how teachers interact with light in classrooms and what the motivations for interactions are.

This paper presents the results of a case-study of interactive lighting in classrooms. In a Danish elementary school, a new lighting system with possibilities of choosing different lighting scenarios was installed in three case-study classrooms. An analysis of the classroom environment was carried out in 2016, prior to the evaluation, investigating the teachers' and students' needs and the effects of the existing lighting on the students' behavior [10]. Based on this analysis, four different dynamic lighting scenarios were defined, which were subsequently implemented in the classrooms and evaluated as the last test phase of the research project in 2017. It was hypothesized that the lighting scenarios would support the teacher in structuring the teaching.

The objective of this paper is to present and discuss the results of how dynamic lighting can be used as a tool for teachers to "set the scene" and thereby support different learning activities by asking: Is there a relationship between the teachers' usage of lighting and the classroom activities? Moreover, what motivations are indicated for using the lighting scenarios?

\section{Methods and Materials}

The research focuses on light as experienced in the classroom, meaning that both the daylight and the electrical light are considered together. A field study was carried out using a mixed-methods approach $[11,12]$ combining quantitative data with qualitative data, creating a holistic understanding of the teachers' interaction with the lighting. While the non-participatory observations and structured datalog information of the lighting control system provided a view of the teachers' interactions with the new lighting system, the interviews provided an insight into the teachers' personal experience.

\subsection{Research Setting and Lighting Scenarios}

The field study setting consists of three classrooms with windows on one side of the room. The renovation of the rooms replaced florescent tube ceiling luminaires with controllable LED lighting. The lighting scenarios were designated as Standard, Smart Board, Fresh, and Relax. 

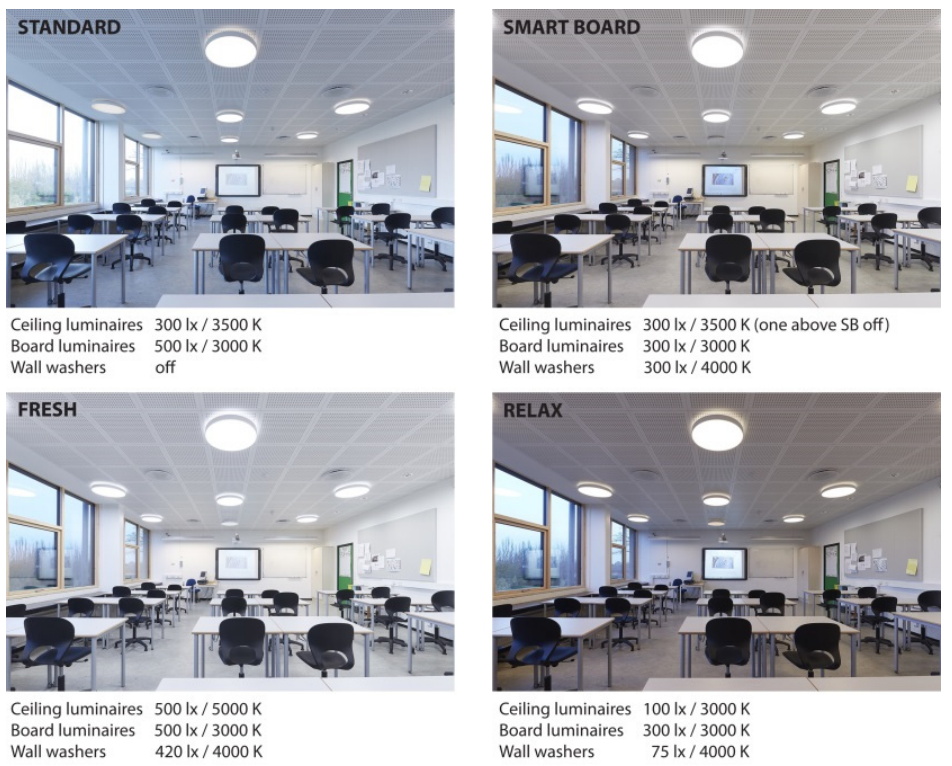

Figure 1. The illuminance level and correlated color temperatures of different luminaire groups used in the four lighting scenarios

Teachers could select these scenarios, or switch off the lights entirely, as well as manually adjust the CCT (Correlated Color Temperature) and illuminance (lux) of each scenario. The Standard scenario was designed to fulfill the requirements of standard DS/EN 12464-1 DKNA. The primary aim of the Smart Board scenario was to prevent the artificial lighting from reducing the contrast of the projected image, yet at the same time to allow the students to perform tasks on their desks. The Fresh scenario was designed to "freshen up" the students, and to increase their alertness while focusing on the teacher or on the task. Finally, the Relax scenario was designed to create a relaxing and informal atmosphere in the classroom by providing dim and warm lighting.

\subsection{Data Collection and Analysis Methods}

The data was collected over a 3.5-month period from 4. September to 15. December and consisted of quantitative data from the lighting system's datalog, and qualitative data from interviews and observations conducted over two shorter periods consisting of a few weeks each. The participants were teachers and school children of three classes, X, Y and Z. Each classroom consisted of 22 to 24 children in the age group 11 to 12. The three classes had each a main teacher, all female, with an additional five rotating teachers, two females and three males. With the exception of one female, who was in her fifties, all the teachers were in their thirties. 


\subsubsection{Quantitative data: Lighting control data log}

Quantitative data was collected from the lighting system's datalog over the period $04 / 09 / 2017-15 / 12 / 2017$ in which absent data, day trips and holidays were left out of the analysis. The datalog tracked the choice of lighting scenario and manual adjustments of the lighting. The analyses used the durations of lighting scenarios, calculated in minutes, as well as the instances of choice, calculated as the number of times a setting occurred. The break periods were not factored into the analysis of durations, but they were included in the count of instances. The total count was plotted against week numbers to assess the trend of usage. Due to holidays, absence from classrooms and missing data, the week 42 and some single days have been left out of the analyses.

\subsubsection{Qualitative data: Interviews and Observations}

The first observations were carried out in 2017 in weeks 37 and 38, the second round in weeks 48, 49 and 50. The observers focused on specific themes that were derived from the pre-refurbishment observations, but with the option to add additional information if relevant. Whereas the datalog provided information of the teacher's interactions with the lighting system, the observations elucidated the reasons and motivations for their choice of scenario and how this affected the students' behavior. The focus of the observations was on the teachers' interaction with the lighting during their lessons, and on mapping activities taking place and the students' behavior.

Semi-structured interviews [12] were conducted during September and repeated again in December with the same teachers to track their progress and experiences. These interviews addressed the teachers' experience with the new lighting, their motivations for using the scenarios, the changes in students' behavior, and how they adapted the lighting to their teaching strategies. Within the span of research, eight teachers were observed, and six participated in group interviews which were conducted with two and four teachers at a time. Group interviews (20-30 minutes) were conducted to allow the teachers to share their usage of the scenarios and experiences with each other, generating a discussion [12].

\subsubsection{Combining Quantitative and Qualitative data}

The data from the observations were thematised through content analysis [12] into the main categories of the teaching and learning activities and organized into timelines, specific to each teacher, where their lighting scenario choices were added from the data $\log$. Within this, the durations of each lighting scenario and a certain activity could be counted side by side. These timelines enabled a comparison of the teachers' individual patterns of usage of lighting scenarios and their corresponding activities. The timelines of three teachers (T1, T2 and T3) were chosen for further analysis, and the relative amount of each lighting situation for certain activities were calculated in percentages. The choice of these teachers was based on the fact that their lessons had been observed both in the autumn and winter, and their lessons contained all the activities of interest. While explaining the findings from the analysis of the interviews, some examples of also other teachers' habits of using lighting as a tool have been presented. 


\section{$3 \quad$ Results}

On average, lighting scenarios were chosen four times a day in a classroom, including the option to switch lights off and adjust them manually. Only lessons before lunch were included in the count. The usage rose in the beginning and came gradually down to a steady level, but peaked in the last week. The increased use of the lighting scenarios in the first weeks of the study was due to a curiosity towards the new technology and a will to play with it, after which the novelty wore off. This was equally reflected in the interviews by a teacher: "I am not aware of the light anymore. In the beginning it was exciting, but light does not play as big role now. [...] There are 10.000 other things" [T2: 18/12/2017]. Deciding a scene was not an automatic part of every teaching routine, nor their first priority. Rather, the teachers had to adapt the lighting scenes to their teaching strategies, students' needs and lessons.

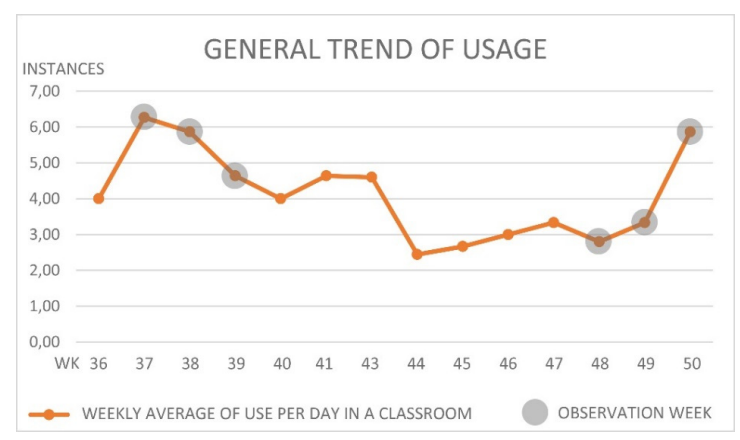

Figure 2. Weekly averages of daily use of lighting scenarios in a classroom. Repetitive rapid changes of lighting with only $1-60$ seconds interval were counted as one instance.

The analysis identified that the usages of lighting scenarios was related to five different types of motivations.

\subsection{Supporting and structuring learning activities}

The analysis of the classroom activities and teachers' usage of lighting scenarios in parallel, indicate that the change of a lighting scenario often happened with a change in activity during the lessons. This was confirmed in the interviews with T3 who explained that the choice of lighting depended on the learning situation. On the other hand, T4 explained that he only changed the lighting to suit the activity when he entered the classroom, and mostly only changed this during the lesson if they had to watch a video on the Smartboard, or if someone had changed the lighting while he was out of the class. A general finding which emerges from the data is the individual nature of interaction that the teachers have with the lighting. A good example is the reading session (Figure 3), which involved students either reading themselves or the teacher reading aloud. The graph indicates that T1 preferred to use the Relax scenario, whereas T2 only used the Fresh scenario and T3 mostly used the Standard scenario. T3, however, changed his preference towards the end of the year and started to use the Relax scenario for the Christmas stories. 


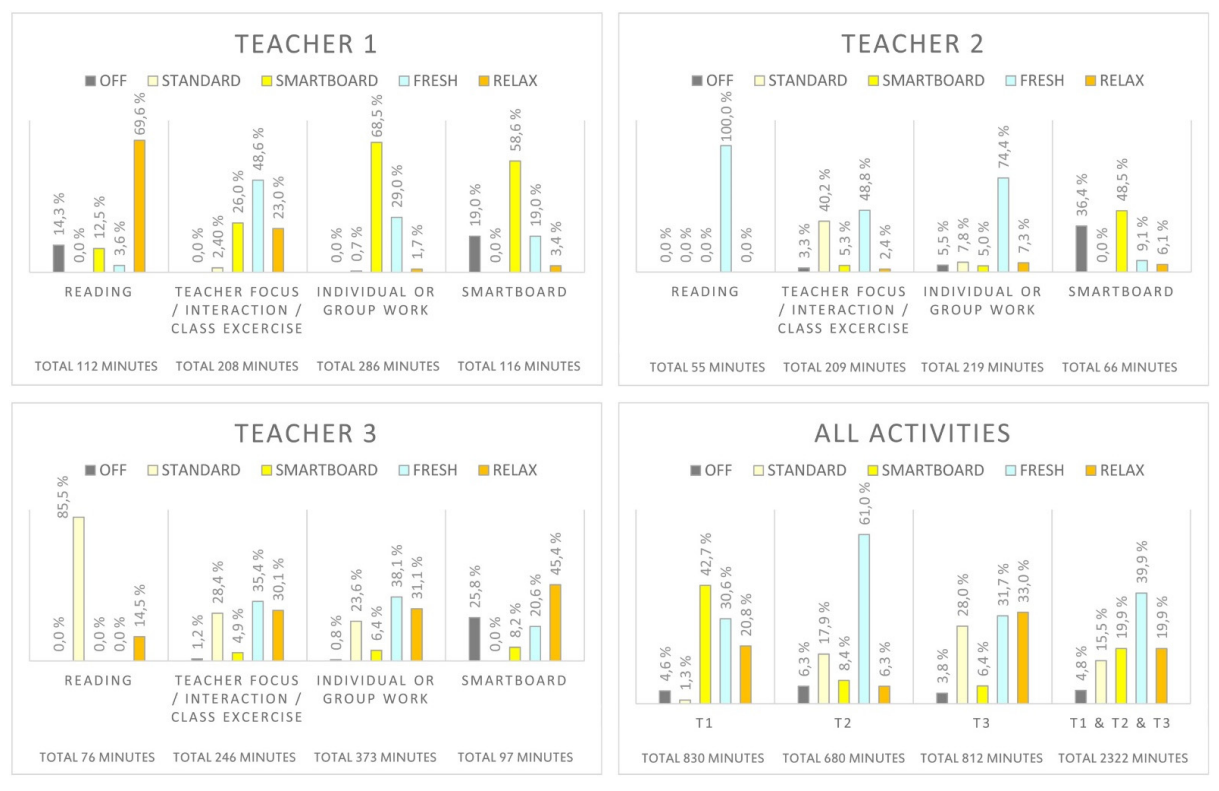

Figure 3. Relative amounts of lighting scenarios used by teachers during selected activities and all activities

In the interview, $\mathrm{T} 3$ explained that his choices were related to a difference in learning situation. During the subject of religion, the students are allowed to leave the classroom to work on their assignment somewhere else, and come in again, which can lead to lots of interruptions. In these situations, T3 preferred to use either the Standard or the Fresh scenarios, as he found them activating. He explained that the Relax scenario, which he uses during mathematics during which the students remain in the classroom working either alone or in groups, would be ill-suited for these kinds of active sessions, because the students have adapted to the idea that mathematics entails a calm uninterrupted learning environment. The teacher described the interactive relation of learning activities and lighting as followed: "Each activity has their scenarios. [...] the [learning] scenarios influence the lighting and the lighting influences [learning] scenarios to take place." [T3: 15/12/2017]. Besides the motivation of supporting a certain activity with an adequate lighting scenario, there was equally an incentive to use lighting as a tool to structure the learning activities. In the interview, T3 explained that he also uses the lighting to signal the start of a new activity, for example, when they need to read. As such, the choice of lighting scenarios depended on the learning activity and not so much on the subject being taught. T3 also referred this to the new pedagogical approach in teaching which promotes dynamism in school work with changing activities and working methods: "A lot of the improvement results in a teaching that becomes more dynamic and is not uniform and boring for the children. That you also are able to change the lighting scenes at the same time, do not result in that the children sit still that day, and it is not the great acoustic panels that make the children sit calm, but it means that the children will have some of these funny changes [during the day] and that matters." [T3: 15/12/2017] 


\subsection{Communicating with lighting and involving students}

The example of T3 who uses light to signal a change of activity shows that lighting can be used as a tool to communicate with students. During the September observations T4 had a similar manner of structuring lessons with lighting changes. He additionally indicated his lighting choice verbally to the students in order to create a cue for changing activity. Based on both the observations and the interviews, it could be stated that teachers involved students in their lighting interaction and asked their opinion about which lighting scenario would suit them. Often students made unsolicited comments about the lighting as well as requests, to which the teachers responded by changing the lighting. After becoming accustomed, the students would sometimes remind the teacher to change the light for a certain activity if they forgot.

\subsection{Affecting the students' activity level and behavior}

Lighting was also used with the intention to influence the students' activity levels and behavior. For instance, T4 used the Fresh scenario as a means to indicate to the students that they should focus. T2 and T5 related their lighting interaction to the energy levels of students: they told that if the classroom was too energized, they would switch to the Relax scenario to tone it down which, according to their experience, worked. T3 said that he did not use the lighting to make students calmer when they were noisy and explained that the students only see the light and not hear it, and that only what they heard could stop them from off-topic behavior. In addition, the interviews in December with T3 and T4 indicated that it was very difficult to see what the effect of lighting was, and what the effect of other factors were in the refurbished classrooms such as for example the new acoustic panels. In addition, the students had matured by one year, and there had equally been a change in the way the groups were formed.

\subsection{Creating atmospheres}

One specific motivation in using lighting as a tool was to create an adequate atmosphere for the learning situation or activity, which sometimes related to the maturity level of the group. T3 explained in the second interview that during the Christmas reading sessions, when reading the story to the class, he chose the Relax scenario and manually adjusted it to make it even dimmer with the intention to create a cozy atmosphere. In the second interview, T4 explained that he considered the maturity level of the class rather than the specific learning situation. For this, warm toned light was chosen to create a cozy atmosphere, thus a comfortable learning environment for the students who were lagging behind. T4 used the manual adjustment to try and create a lighting situation with sufficient amount of illuminance but with a warm CCT, as this kind of scenario was missing in the choices. The analysis revealed a seasonal variation with respects to the preferred scenarios. When approaching the winter, the preferred CCT shifts towards warmer tones, and illuminance levels decrease as the natural light coming from outdoors becomes scarcer. As an example, the use of the Relax scenario increases. Both T3 and T4 explained in the winter interviews that their usage had changed towards warmer settings, which they sometimes tried to manually adjust to even lower settings. T4 related this choice of warm scenario to the situation of natural light outside, 
and to the high contrast between inside and outside which occurs during the dark season. Although it was outside the scope of this study, this relation between the use of scenarios and seasonally changing daylight conditions is an interesting preliminary finding and worth studying more accurately and with the use of statistical methods.

\subsection{Supporting visual tasks and visual comfort}

Finally, teachers interacted with lighting in order to create suitable lighting conditions to support visual tasks and to maintain visual comfort. Most of the time this was achieved by using the manual override of shades or by drawing the curtains to reduce glare from the direct sun light, or to increase the visibility of the smartboard.

The graph in Figure 3 illustrates that the Smart board scenario was used prominently when the smart board was used as part of the activities. However, in the interviews the teachers were critical about this scenario. Several teachers mentioned that the scenario was not sufficient enough for smart board visibility: in the programming, all the round ceiling lights should have been turned off and not only the middle light fixture. Teachers had solved the visibility issue by either choosing the Relax scenario (T3), which generally has lower lighting levels, or by turning the lights off (T2, T4).

\section{Discussion \& Conclusion}

By applying mixed methods, the findings of this study elucidate how the visual effects related to coziness and visibility play a large role in both the teachers' and students' sense of well-being and comfort. Whereas preferences of lighting scenes have previously been studied in lab-tests [2], this study contributes to the existing field [2, 4, 5, 14 ] by conducting it in a field-study where daylight is a co-founding factor.

In the preliminary study prior to the refurbishment, the light was subconsciously a part of the teachers' everyday practice of the classroom. By contrast, the evaluation of the teachers' interaction with the dynamic lighting revealed that the teachers had become aware of the possibilities and their usage of the lighting.

A comparison between the chosen lighting scenarios and the learning activities revealed a relation between them, indicating that teachers used lighting scenarios as a tool to both support activities and structure lessons through changes in lighting. However, the manner in which the lighting scenarios were used i.e. which scenario for which activity, was individual and teacher specific, and was based on their own pedagogical approaches.

Our sample size in this study was small including three case study teachers and five other teachers which were observed over five weeks; further studies with larger sample of teachers and a longer observation period combined with statistical analysis might also reveal some general patterns and correlations between chosen lighting scenarios and teaching activities, besides the individual patterns.

The analysis of the interviews and observations showed that different strategies were employed. The teachers were aware of which scenes to choose to affect a particular behavior, and according to their experience, lighting had an effect. Additionally, the analysis also showed that the teachers were motivated to affect the students' behav- 
ior throughout the day, by creating a dynamic variation of the lit surrounding to accompany the dynamically changing activities. As the findings show, also seasonal changes are a co-founding factor in the teachers' practice of creating atmospheres. This is seen by the increased use of the Relax scene while reading Christmas stories, or by the amount of times the lighting was switched off while watching Christmas shows.

The observations revealed that the students were partly decision-makers in the choice of lighting scenarios. Either the teachers involved them actively when they chose a scenario, or the students would make unsolicited suggestions. As such the students became co-creators in defining suitable classroom spaces and decision-makers in deciding what they considered as the optimal learning environment for the task. In this way, the four lighting scenarios are a tool that promote the interaction between students and teachers. Becoming participants in the design of learning environments affects motivation to learn: "Improving the congruence between the perspectives of students and those creating the learning environment (i.e., teachers and instructional designers), thus, is likely to improve student learning" [13].

As indicated in the interviews, one of the reasons that the lighting system was used less after the initial period can be related to the busy schedules during teaching sessions. Naturally, lighting is not necessarily the main priority, and was not part of the planning of the lessons but was used rather spontaneously. As such it is worth re-thinking the design by considering a more autonomous lighting system. However, previous research argues that a hybrid between full autonomous systems and manual user control are most desirable, because providing the users with the possibility to manually change the lighting has a positive effect on their well-being and comfort [15]. Therefore, the balance between a complete automated system and interactive control must be considered.

In conclusion, this research has illustrated how lighting can be an integrated part of future pedagogic approaches. Five motivations for using lighting as a tool to support teaching were identified:

- Supporting and structuring learning activities

- Communicating with lighting and involving students

- Affecting students' activity level and behavior

- Creating atmosphere

- Supporting visual task and visual comfort

We argue that these motivations should be considered while designing interactive systems for dynamic control of lighting in classrooms. Results show that teachers generate individual patterns and manners in using lighting as a tool, thus, the systems should allow this versatility. Besides interaction possibilities, a more developed automatization and a self-learning system could ease the use for less-motivated teachers and also take into account the seasonal variation of daylight availability.

These findings raise the issue whether classroom lighting in the future should be designed not only according to the DS/EN standards for Lighting, but to a larger extent aligned with the users' needs for different lighting in a classroom. This could imply for example designing lighting typologies that would enable teachers to orchestrate space and atmosphere in their daily practices with the use of lighting scenarios. This study also elucidates the importance of conducting evaluations after implementations of lighting to create lighting designs that meet users' needs. 


\section{Acknowledgement}

We warmly thank children and teachers of the 6th grade at Herstedlund School and Albertslund Municipality, Zumtobel and Sweco, our project partners in the Interreg project, Lighting Metropolis. Thanks to the Academy of Finland for the funding which enabled H. Pihlajaniemi to work in the project as a visiting researcher at the Aalborg University.

\section{References}

1. Undervisningsministeriet, https://www.uvm.dk/statistik/grundskolen/elever/elevtal-igrundskolen. https://www.uvm.dk/folkeskolen/fag-timetal-og-overgange/undervisningenssamlede-laengde, last accessed 2017/12/14.

2. Hansen, E. K. Louring Nielsen, S. M. Georgieva, D. \& Schledermann., K. M.: The Impact of Dynamic Lighting in Classrooms. A review on Methods. Institute for Computer Sciences, Social Informatics, Telecommunications Engineering 2018. ArtsIT 2017/DLI 2017, LNICST 229, pp. 314-323 (2018)

3. Barkmann, C., Wessolowski N., and Schulte-Markwort, M. Applicability and efficacy of variable light in schools. Physiol. Behav. 105, 621-627 (2012)

4. Mott, M.S., Robinson, D.H., Walden, A., Burnette, J., Rutherford, A.S.: Illuminating the effects of dynamic lighting on student learning. SAGE Open 2012, 1-9 (2012)

5. Sleegers, P.J.C., Moolenaar, N.M., Galetzka, M., Pruyn, A., Sarroukh, B.E., van der Zande, B.: Lighting affects students' concentration positively: findings from three dutch studies. Lighting Res. Technol. 45, 159-175 (2013)

6. Keis, O., Helbig, H., Streb, J., Hille, K.: Influence of blue-enriched classroom lighting on students' cognitive performance. Trends Neurosci. Educ. 3, 8-92 (2014)

7. Choi, K., Suk, H.J.: Dynamic lighting system for the learning environment: performance of elementary students. Opt. Soc. Am. (OSA), 24(10) (2016)

8. Wessolowski, N., Koenig, H., Schulte-Markwort, M., Barkmann, C.: The effect of variable light on the fidgetiness and social behavior of pupils in school. J. Environ. Psychol. 39, 101-108 (2014)

9. BRANZ Ltd.: Designing Quality Learning Spaces: Lighting. New Zealand: Ministry of Education (2007). ISBN 0-478-13619-6; WEB ISBN 0-478-13624-2

10. Georgieva, D. Schledermann, K. M. Louring Nielsen, S. M. \& Hansen, E. K. (2018): Designing User Centred Intelligent Classroom Lighting. Institute for Computer Sciences, Social Informatics, Telecommunications Engineering 2018. ArtsIT 2017/DLI 2017, LNICST 229, pp. 314-323 (2018)

11. Creswell, J.W.: Research Design: Qualitative, Quantitative, and Mixed Methods Approaches, 4th ed. SAGE Publications Inc., USA (2014)

12. Bryman, A.: Social Research Methods, 4th ed. Oxford University Press, New York (2012)

13. Könings, K. D., Seidel, T., \& van Merriënboer, J. J. G. Participatory design of learning environments: integrating perspectives of students, teachers, and designers. Instr Sci, 42(19). (2014)

14. van Someren, K.L., Beaman, C. P. and Shao, L.: Users' experience of lighting controls: A case-study. Lighting Res. Technol. 2017; 0: 1-16 (2017)

15. van Essen H, Offermans S \& Eggen B: Exploring the Role of Autonomous System Behavior in Lighting Control. In: Proceedings of Designing Interactive Lighting workshop at DIS 12, Newcastle, Great Britain (2012) 